\title{
Neural basis of implicit cognitive reappraisal in panic disorder: an event-related fMRI study

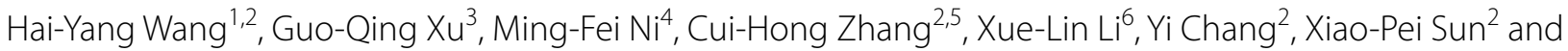
Bing-Wei Zhang ${ }^{2,3^{*}}$ (1)

\begin{abstract}
Background: Panic disorder (PD) is thought to be related with deficits in emotion regulation, especially in cognitive reappraisal. According to the cognitive model, PD patients' intrinsic and unconscious misappraisal strategies are the cause of panic attacks. However, no studies have yet been performed to explore the underlying neuromechanism of cognitive reappraisal that occur on an unconscious level in PD patients.

Methods: Twenty-six patients with PD and 25 healthy controls $(\mathrm{HC})$ performed a fully-verified event-block design emotional regulation task aimed at investigating responses of implicit cognitive reappraisal during an fMRI scan. Participants passively viewed negatively valanced pictures that were beforehand neutrally, positively, or adversely portrayed in the task.

Results: Whole-brain analysis of fMRI data showed that PD patients exhibited less activation in the right dorsolateral prefrontal cortex (dIPFC) and right dorsomedial prefrontal cortex (dmPFC) compared to HC, but presented greater activation in parietal cortex when negative pictures were preceded by positive/neutral vs negative descriptions. Simultaneously, interactive effects of Group $\times$ Condition were observed in the right amygdala across both groups. Furthermore, activation in dIPFC and dmPFC was is negatively correlated to severity of anxiety and panic in PD when negative images were preceded by non-negative vs negative descriptions.

Conclusions: Emotional dysregulation in PD is likely the result of deficient activation in dIPFC and dmPFC during implicit cognitive reappraisal, in line with impaired automatic top-down regulation. Correlations between severity of anxiety and panic attack and activation of right dIPFC and dmPFC suggest that the failure to engage prefrontal region during implicit cognitive reappraisal might be associated wtih the severity of anxiety and panic; such functional patterns might be the target of possible treatments.
\end{abstract}

Keywords: Panic disorder, Emotion regulation, Implicit cognitive reappraisal, fMRI, Prefrontal cortex

\section{Background}

Emotional regulation is defined as an attempt to influence the emotions of oneself or others [1]. Negative emotions caused by adverse events need to be regulated to avoid interfering with ongoing activities and long-term goals

\footnotetext{
*Correspondence: zhbw@dmu.edu.cn

${ }^{2}$ Department of Neurology and Psychiatry, The First Affiliated Hospital of Dalian Medical University, No.222, Zhongshan Road, Dalian 116011, Liaoning Province, China

Full list of author information is available at the end of the article
}

[2]. Successful control of unpleasant emotion is critical to an individual's well-being, health, and psychological and social functioning [3]. Emotion dysregulation (difficulties in effectively managing one's emotions) is closely associated with the onset, maintenance, and therapy of various types of anxiety disorders [4].

Panic disorder (PD) is an anxiety disorder characterized by the recurrence of spontaneous panic attacks with psychological, physical, and functional conditions [5]. Clinically, emotion dysregulation is considered to be a pivotal aspect in the pathophysiological mechanism of

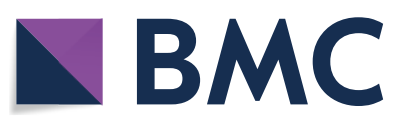

(c) The Author(s) 2021. This article is licensed under a Creative Commons Attribution 4.0 International License, which permits use, sharing, adaptation, distribution and reproduction in any medium or format, as long as you give appropriate credit to the original author(s) and the source, provide a link to the Creative Commons licence, and indicate if changes were made. The images or other third party material in this article are included in the article's Creative Commons licence, unless indicated otherwise in a credit line to the material. If material is not included in the article's Creative Commons licence and your intended use is not permitted by statutory regulation or exceeds the permitted use, you will need to obtain permission directly from the copyright holder. To view a copy of this licence, visit http://creativeco mmons.org/licenses/by/4.0/. The Creative Commons Public Domain Dedication waiver (http://creativecommons.org/publicdomain/ zero/1.0/) applies to the data made available in this article, unless otherwise stated in a credit line to the data. 
anxiety and mood disorders and is a hallmark of PD [6, 7]. Indeed, cognitive emotion dysregulation as an important mechanism in PD is increasingly receiving attention [8-10].

Based on the emotion regulation model proposed by Gross [11], cognitive reappraisal, the most extensively explored skill is one type of antecedent-focused emotion regulation strategy that alters the trajectory of emotional responses by changing the meaning of the situation [12]. Reinterpretation and distancing are two cognitive reappraisal tactics [13]. Reinterpretation is described as altering one's interpretation of the stimulus or situation that provokes the emotion, whereas distancing is described as altering one's personal or psychological distance from the stimulus or situation that elicits emotion. Cognitive reappraisal is remarkably effective in emotion regulation strategies [11] and results in enduring effects [13]. Decreased use of cognitive reappraisal strategies explains some catastrophic interpretations of anxiety-inducing conditions (for example, "catastrophic thinking"), which serves a critical role in the theory of cognition in PD [14, 15]. Based on PD's cognitive model, panic is attributed to catastrophic misappraisal of bodily sensations [16], referring to patients' tendencies to catastrophically misunderstanding psychosomatic responses that are associated with reduced use of cognitive reappraisal, which can act as a conditioning stimulus to trigger and sustain panic [17]. Emotion regulation strategies have been measured using emotion regulation questionnaires; for instance, our previous research showed that PD patients may involve emotion dysregulation associated with cognitive reappraisal [18]. We found that PD patients use fewer positive reappraisal but more catastrophic strategies compared to healthy controls [18]. Therefore, it has crucial theoretical and practical significance to explore the neural mechanisms of cognitive emotion dysregulation in patient with PD.

Besides cognitive reappraisal, another emotion regulation strategy involving Gross's emotion regulation model is particularly relevant to PD is expressive suppression, a less well studied. Expressive suppression is a responsefocused maladaptive strategy that involves consciously inhibiting behavioral responses to emotions [3]. Patients with PD commonly attempt to hide their anxiety symptoms by using maladaptive expressive suppression strategy to control their psychosomatic responses, fearing others may notice them, which is unsuccessful and frequently contributes to additional deterioration of symptoms [19]. While some evidence suggests that patients with PD use more expressive suppression strategy compared to the healthy controls $[9,20]$, one study failed to detect this difference [21]. Thus, there is no consistent conclusion about the differences in the use of expressive suppression between PD and healthy control compared to cognitive reappraisal.

The process of emotional regulation can be either explicit (conscious) or implicit (unconscious) [22, 23]. In clinical practice, panic attacks are suddenly and sometimes unexpectedly paroxysmal bursts of severe anxiety [24]. Catastrophic misappraisal can be subconsciously manipulated, such as during panic attacks while sleeping or when particular catastrophic notions are unrecalled [24], and there is usually no conscious cognitive reappraisal process before or during panic attacks. Studies that have examined the mechanisms of anxiety psychopathology show that the emotional regulation difficulties in anxiety are essentially due to deficient engagement of implicit emotion regulation strategies [25]. The catastrophic cognition hypothesis states that PD patients unconsciously evaluate internal and external stimuli that are inadequate to trigger a violent response as threat signals, consequently triggering panic attacks [16]. Recent studies have found that PD patients demonstrated an anomalous mismatch negativity of acoustic and visual (emotional and non-emotional) stimuli, implicating anomalous implicit information processing in patients with PD [26, 27]. Therefore, we speculate that it is likely that PD patients' intrinsic and implicit cognitive reappraisal strategies are responsible for triggering panic attacks and that PD-related abnormal emotional regulation is not only manifested in the realm of consciousness but likely more in implicit reappraisal mechanisms.

Over the last decade, studies investigating the neural mechanism that contribute to emotion dysregulation in PD have shown consistent hypo-activation in prefrontal regions involving the dlPFC, $\mathrm{dmPFC}$, and ventrolateral prefrontal cortex during cognitive reappraisal $[8,9]$. Patients in these researches were asked to interpret the aversive stimuli as less negative deliberately and consciously through reinterpretation to reduce their unpleasant emotional experiences. Thus, the extra effort and cognitive control might indicate the possibility that participants had to deliberately produce their substitute explanations for the aversive pictures. However, these studies do not effectively reveal the neural basis of cognitive reappraisal that occurs out of consciousness in PD.

Zhang et al. adopted an implicit reappraisal paradigm to explore the neural basis and time processes in unconscious reappraisal in PD with event-related potentials (ERPs) [10, 28]. In Zhang's study, PD patients and healthy controls received a brief neutral or negative description before viewing the negative pictures. During passive viewing of adverse pictures, cognitive emotion regulation processes were effortless, uninstructed, and unconscious [23]. The results showed that the negative pictures predescribed with neutral descriptions exhibited decreased 
late positive potential (LPP) amplitudes than the negative pictures pre-described with a negative description in healthy subjects. In contrast, no reliable effect of description condition was found for LPP in the PD patients. These results demonstrate an impairment in the regulatory process of cognitive reappraisal in PD [10]. Moreover, the task used is a reactive reappraisal paradigm that avoids the effects of increased task effort or additional underlying emotional processes, providing further proof that reduced LPP modification supports aberrant implicit cognitive reappraisal in PD patients [28]. ERP can effectively reflect the time course of brain electrical activity, but the low spatial resolution means that it can be difficult to pinpoint the precise localization of functional neural correlates. In contrast, fMRI has high spatial resolution and is the most effective method to study neural functioning. However, to our knowledge, there have been no fMRI studies regarding the nerve mechanism of implicit cognitive reappraisal in PD.

Therefore, the current study was designed to explore the neural basis of implicit cognitive reappraisal in PD by using neuropsychological assessments, self-report measures of emotional experience, and fMRI. The reappraisal tactics for emotion regulation mainly include reinterpretation and distancing [29]. Reinterpretation was used as a cognitive reappraisal strategy in this research based on previous studies $[9,23]$. Behavioral data and fMRI data were recorded from PD and $\mathrm{HC}$ using the implicit cognitive reappraisal paradigm developed by Foti and Hajcak [30]. This task involves two conditions: (a) negative images were pre-described with non-negative (neutral or positive) descriptions (implicit reappraisal condition) and (b) negative images were pre-described with negative descriptions. Studies in implicit emotion regulation of healthy populations have shown that implicit emotion regulation can effectively decrease negative valence ratings of subjects, and increase activity in prefrontal regions such as dIPFC and dmPFC, the parietal lobe, and other brain regions, accompanied by decreased activity in limbic systems [31, 32]. In earlier work, we investigated the neural bases of implicit emotion regulation in healthy subjects by using the implicit cognitive reappraisal paradigm and demonstrated that implicit reappraisal processes could recruit prefrontal areas involving the dIPFC, dmPFC, and parietal cortex to modulate emotional responding [23]. On the basis of these results, we hypothesized that PD patients would not be able to automatically adjust their emotional state through implicit cognitive reappraisal strategy when receiving neutral/ positive descriptions before the negative affective pictures. Moreover, we expected that the PD group would report negative feelings and correspondingly show decreased activity in prefrontal regions involving dIPFC and dmPFC compared to healthy controls. This would reflect the greater affective responsiveness to adverse stimuli and the lack of automatic inhibition of emotional responses to adverse stimuli in PD. Given that the biological model points to inadequate cognitive control as a factor in generating panic [23,33], we further hypothesized that emotion disorder in PD might be the result of inadequate top-down control during implicit reappraisal.

\section{Materials and method}

\section{Participants}

Participants were between the ages of 18 and 65, righthanded, were Chinese Asians (Han nationality, in China only), without current and past major medical or neurological conditions, and certified by two board-certified physicians. Since this is the first investigation to explore implicit cognitive reappraisal in $\mathrm{PD}$, it is unable to determine the required sample size based on a priori power analysis. Therefore, we set the desired sample size to 26 in the PD group with reference to two recent fMRI studies in PD that explore deliberate cognitive reappraisal strategy $[8,9]$. Twenty-six untreated patients met criteria for clinically predominant PD based on Diagnostic and Statistical Manual of Mental Disorders, 5th Edition (DSM-5) [5] and $25 \mathrm{HC}$ without DSM-5 axis-I history were recruited from the emergency and outpatient departments of the First Affiliated Hospital of Dalian Medical University and the surrounding communities. After providing written informed consent, participants completed the Hamilton Anxiety Rating Scale (HAM-A) [34], Hamilton Depression Rating Scale (HAM-D) [35], the Panic-Associated Symptom Scale (PASS) [36], Panic Disorder Severity Scale (PDSS) [37], and Cognitive Emotion Regulation Questionnaire (CERQ) [38]. Individuals were informed of the safety and eligibility criteria for fMRI scan: no cognitive impairment (dementia, traumatic brain damage, mental deficiency, and instrumental brain syndrome), no other neurological impairment, and no contraindications to fMRI (e.g., implanted ferrous metal, pregnancy, claustrophobia). Considering the variability in the treatment process, all subjects were free of any antipsychotic drugs for at least two weeks (benzodiazepine intake were medication-free $48 \mathrm{~h}$ ) before the scan. Owing to the high co-morbidity rate in PD and affective or other anxiety disorders [39], co-morbid mood and other anxiety disorders were permitted if the PD diagnosis was primary. In the PD group, two patients were combined with generalized anxiety disorder, four patients with social anxiety disorder, and three patients with depressive disorder. Table 1 presents the demographic characteristics of the 51 participants. Informed consent was obtained from all participants prior to participation according to the Declaration of Helsinki principles. This study was approved 
Table 1 Demographics and clinical features of participants

\begin{tabular}{lllll}
\hline & PD & HC & $\boldsymbol{F}_{1,49} / \mathbf{X}^{2}$ & P score \\
\hline Gender (male/female) & $13 / 13$ & $12 / 13$ & $\mathbf{X}=0.20$ & 0.89 \\
Age (years) & $35.6(8.0)$ & $35.2(6.7)$ & 0.033 & 0.86 \\
Education in years & $13.0(3.1)$ & $14.4(2.0)$ & 3.4 & 0.072 \\
HAM-A & $15.3(6.3)$ & $2.3(2.1)$ & 94.4 & $<0.001^{* * *}$ \\
HAM-D & $10.8(5.2)$ & $3.5(1.8)$ & 4.29 & $<0.001^{* * *}$ \\
PDSS & $10.3(5.0)$ & - & - & - \\
PASS & $8.5(4.7)$ & - & - & - \\
CERQ scales & & & & \\
Self-blame & $6.2(2.3)$ & $5.3(1.7)$ & 2.25 & 0.140 \\
Acceptance & $7.3(1.9)$ & $6.48(1.7)$ & 2.50 & 0.120 \\
Rumination & $7.7(1.6)$ & $6.4(1.8)$ & 6.92 & $0.011^{*}$ \\
Positive refocusing & $4.8(1.2)$ & $5.3(1.6)$ & 3.87 & 0.055 \\
Refocus on planning & $7.1(2.1)$ & $7.5(1.7)$ & 2.95 & 0.092 \\
Positive reappraisal & $6.2(1.6)$ & $7.5(1.4)$ & 9.84 & $0.003^{* *}$ \\
Putting into perspective & $5.6(2.0)$ & $6.9(1.4)$ & 9.40 & $0.004^{* *}$ \\
Catastrophizing & $5.7(2.0)$ & $3.4(1.3)$ & 25.49 & $<0.001^{* * *}$ \\
Other-blame & $5.0(1.7)$ & $4.6(1.4)$ & 0.57 & 0.453 \\
\hline
\end{tabular}

$P D$ panic disorder, $H C$ healthy controls, HAMA Hamilton Anxiety Rating Scale, HAMD Hamilton Depression Rating Scale, PDSS Panic Disorder Severity Scale, PASS Panic-Associated Symptom Scale, CERQ Cognitive Emotion Regulation Questionnaire

${ }^{*} P<0.05,{ }^{* *} P<0.01,{ }^{* * *} P<0.001$

by the ethics committee of the First Hospital of Dalian Medical University.

\section{Measures}

The PASS is a nine-item scale used for evaluating the severity of a wide range of symptoms of PD [36]. The symptoms range from the frequency and intensity of situational panic attacks, the frequency and intensity of spontaneous panic attacks, the amount and intensity of anticipatory anxiety, to phobia-induced distress. The total scores range from 0 to 28 . The Chinese version of the PASS scale was used, which has high internal consistency (Cronbach's $\alpha=0.72$ ) and reliability (test-retest intraclass correlation coefficient $=0.64-0.77$ ) [40] .

The Panic Disorder Severity Scale (PDSS) is a sevenitem scale to evaluate the overall severity of panic disorder [37]. The scale evaluates the frequency of panic attacks, distress during attacks, anticipatory anxiety, fearful avoidance, feelings of fearful avoidance, the impairment in functional ability at work, and the impairment in social functioning. It is a four-point Likert scale format with a total score between 0 and 28. The Chinese version of the PDSS scale has high internal consistency (Cronbach's $\alpha=0.74$ ) and reliability (test-retest intraclass correlation coefficient $=0.70-0.89$ ) [40].
The HAM-A is a 14-item questionnaire administered by clinicians to measure the severity of anxiety symptoms [34].Severity of depressive symptoms was measured using the validated 24-item Hamilton Depression Rating Scale (HAM-D) [35].

The CERQ was used to assess cognitive emotion regulation strategies after exposure to unpleasant life incidents [38]. The CERQ is composed of 9 distinct scales, all consisting of two items, including maladaptive strategies (i.e., rumination, self-blame, catastrophizing, and otherblame) and adaptive startegies (i.e., acceptance, positive refocusing, refocus on planning, positive reappraisal, and putting in perspective). Each item was scored on a 5-point Likert scale ranging from 1 (almost never) to 5 (almost always). The Chinese version of the CERQ has favorable reliability and validity with Cronbach's $\alpha$ of 0.81 for the full scale, inter-item correlation coefficients of 0.1 for the full scale, and mean inter-item correlation coefficients ranging from 0.19 to 0.71 for each subscale [41].

\section{Stimuli and task}

In the present study, reinterpretation rather than psychological distancing was used as the reappraisal tactic. Participants completed the implicit cognitive reappraisal paradigm developed by Wang et al. [23] during an MRI scan. The paradigm comprised two runs. Each run paradigm comprised 25 trials. The negative pictures were shown for $4 \mathrm{~s}$ in each 16-s trial, preceded and followed by the corresponding dislocated pictures (and fixation crosses in black) for a 12-s total (Fig. 1). In each run, a 3-s scrambled picture first appears on the screen, in which appears either a neutral/positive or negative depiction of the upcoming picture that is still present on the monitor. After 1-3 s (jittered) of presentation of the same scrambled picture with a fixed cross as a baseline, the negative picture was shown for $4 \mathrm{~s}$ and the participant looked at the picture passively. Following this, participants were again shown the dislocated pictures and fixed crosses for $3 \mathrm{~s}$ and were asked to use the keyboard to assess their emotional valence from 1 (none too negative) to 4 (extremely negative). The same dislocated picture with a black fixed cross was then presented for 3-5 $\mathrm{s}$ (jittered) as a baseline. Of interest to our hypothesis was the $4 \mathrm{~s}$, during which subjects passively viewed unpleasant pictures.

We chose 60 negative pictures from the International Affective Picture System [42] as stimuli, using a pixellevel dislocated version of each picture as a baseline. The preceding descriptions of 25 pictures highlighted the negative sides of the pictures (negative descriptions preceding negative pictures condition, NEG-DESC), while the other 25 descriptions depicted pictures with a neutral or positive form (non-negative descriptions preceding negative pictures condition, NNEG-DESC). As a 


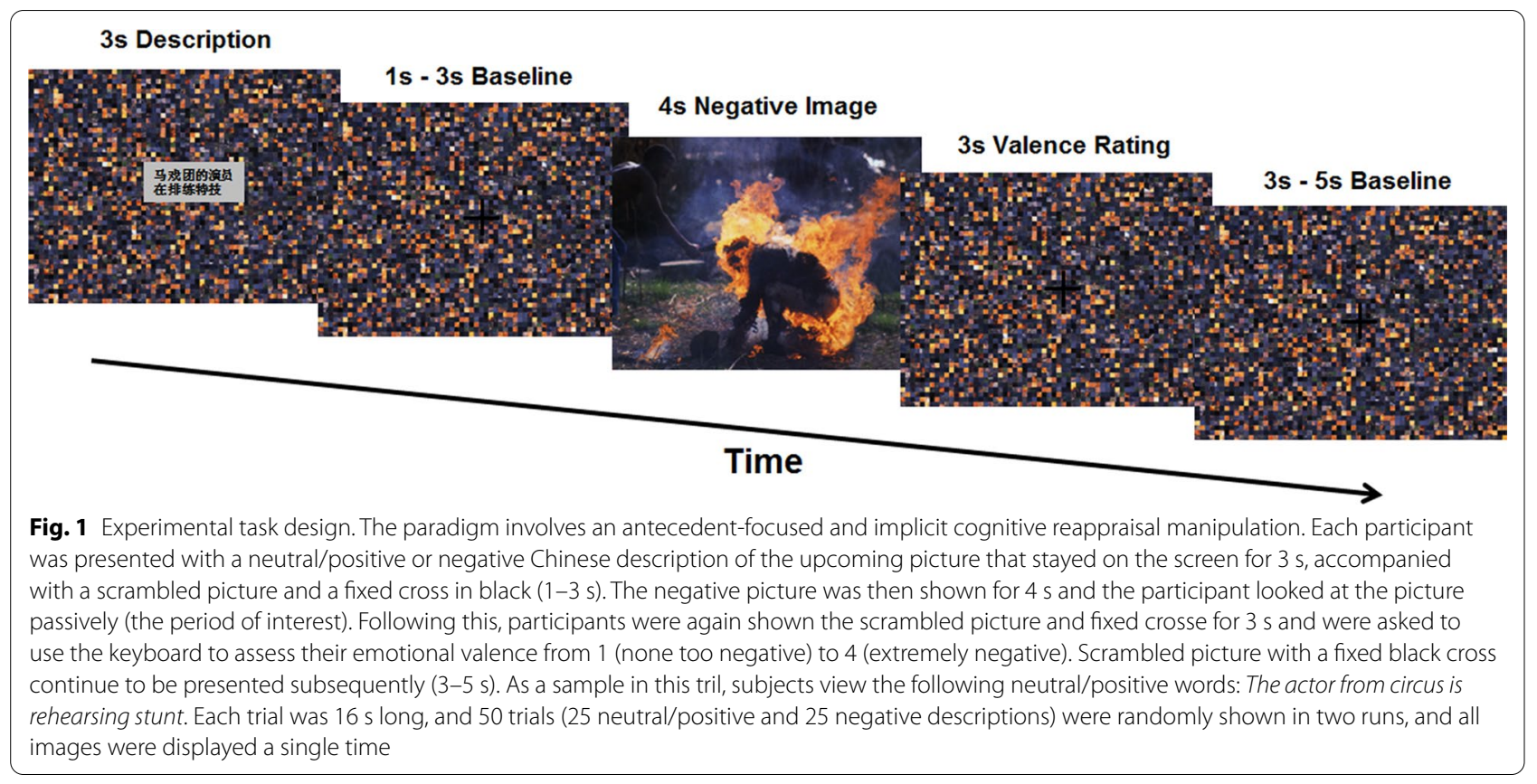

sample, subjects view the following negative descriptive words: This man was burned alive. The same picture's neutral or positive descriptive words were: The actor from circus is rehearsing stunt. The aim of this operation was to investigate the differences in brain activation to negative pictures between the NNEG-DESC condition and NEG-DESC condition. In both runs, the order of trials and the corresponding negative or non-negative descriptions before each negative picture is randomized. All subjects were required to finish 25 NNEG-DESC trials and 25 NEG-DESC trials. Prior to the fMRI scan, participants finished 10 practice trials in which no pictures were used in experimental trials to confirm understanding. Before the task started, subjects were informed to simply watch and do not think otherwise. After the task, participants all reported that they were not aware of the intention of the task was emotion regulation before it started and after it ended and carefully followed the task requirements without doing active thoughts during the task.

\section{Behavioral data analysis}

The behavioral data (i.e., four-point rating of valence) were analyzed using a $2 \times 2$ mixed measures analysis of variance (ANOVA), including the between-subject factors of group (PD, $\mathrm{HC}$ ) and within-subject factors of condition (NNEG-DESC, NEG-DESC). Post hoc pairedsamples $\mathrm{t}$-tests were performed to investigate the presence and direction of group differences in negative affect valence ratings in each condition.

\section{Image acquisition}

Images were obtained using 3.0-Tesla MR scanner (SignaHDx, GE Healthcare, Chicago, IL). A volume head coil was used for radiofrequency reception and transmission. Two fMRI runs sensitive to blood oxygenation level-dependent (BOLD) were performed, lasting $6 \mathrm{~min}$ each. The scanning parameters were as follows: $\mathrm{TR} / \mathrm{TE}=2000 / 30 \mathrm{~ms}$, slice thickness $/ \mathrm{gap}=2.6 / 1.4 \mathrm{~mm}$, slice number $=36$, field of view $(\mathrm{FOV})=220 \times 220$ $\mathrm{mm}^{3}$, matrix size (per slice) $=64 \times 64$, flip angle $=90^{\circ}$. High-resolution T1-weighted anatomical images were collected using BRAVO sequence TR/TE $=8 / 1 \mathrm{~ms}$, FOV $=256 \times 256 \mathrm{~mm}^{2}$, flip angle $=12^{\circ}$, slice thickness $=1 \mathrm{~mm}$, number of slices $=184$, no gap, during the same session for normalization, co-registration, and data visualization.

\section{fMRI data analysis}

Functional MRI data were pre-processed and analyzed using the Analysis of Functional NeuroImages (AFNI) software package [43]. To allow for magnetization equilibrium before image acquisition, the first four volumes of the functional images were discarded. All functional images were slice time corrected with reference to the first acquired slice. Then, a six-parameter rigid-body spatial transformation was used to spatially correct the image for head movement. The high-resolution anatomical images were then acquired for co-registration with the functional scans. Spatial smoothing of the 
normalized functional images was performed with an 8-mm FWHM Gaussian kernel. Finally, linear and quadratic trends were modeled for each voxel's time course to control.

Individual-level whole-brain general linear model analysis was conducted on the preprocessed fMRI data. The regressors of interest were NNEG-DESC and NEGDESC conditions (i.e., corresponding dislocated picture with the fixed cross). Other regressors of non-interest included two regressors of participants viewing picture descriptions and six regressors head movement. The predicted activation time course was convolved with each subject's estimated hemodynamic response function modeled as a gamma probability density function.

Contrast maps for each subject were then resampled with the functional data resolution, normalized to Talairach coordinates. The main aim of our study was to separate emotional responses from implicit cognitive reappraisal; thus, in the presence of negative pictures during NNEG-DESC and NEG-DESC, neural activity was modeled separately, with each item contrasted with the baseline. In particular, the comparison of interest was NNEG-DESC and NEG-DESC. To examine the effects of interest, we carried out three t-tests across participants and produced the following t-maps: the condition effects in HC group ((NNEG-DESC)-(NEG-DESC)), the condition effects in PD group ((NNEG-DESC)-(NEG-DESC)), and the interactions between condition and group (HC ((NNEG-DESC)-(NEG-DESC))-PD ((NNEG-DESC)(NEG-DESC))). Correction for multiple comparisons of contrast maps was performed in the whole brain level with the updated AFNI function 3dClustSim [44], which resulted in a whole-brain corrected probability of $p<0.05$. We set the threshold at 0.05 for corrected results and got the cluster size of 162 through 3dClustSim. Cross-subject alignment [45] was not used as the implicit cognitive reappraisal paradigm was verified $[23,30]$ and the current task had some limitations (e.g., number of trials).

Considering the abundant evidence for amygdala involvement in the encoding of emotional memories and in the perception and labeling of unpleasant stimuli [13], we defined two spherical regions-of-interest (ROI) (radius $=6 \mathrm{~mm}$ ). Peak voxels of the bilateral amygdalae were selected according to previous studies [12, 44]. We converted these coordinates used for ROI selection, given in the literature in MNI space, to Talairach space by a nonlinear transform. These ROIs (right amygdala coordinates: $x=24, y=-3, z=-13$; left amygdala: $x=-16$, $y=-6, z=-10)$ were obtained from previously published studies. After extracting mean beta values from these ROIs of the NEG-DESC and NNEG-DESC conditions from each participant, values were entered into ANOVA to explore main effects and interactions.
Using Pearson's correlations, we conducted a post hoc analysis to investigate a possible relationship between self-reported valence ratings and standardized betas (extracted from significant clusters) in the condition of NNEG-DESC, NEG-DESC, and NNEG-DESC vs NEGDESC within PD and HC groups. To explore the potential connections between important clusters of activation during cognitive reappraisal (NNEG-DESC versus NEGDESC) and symptom severity obtained from questionnaires mentioned above, we also calculated Pearson correlation between mean values extracted from significant clusters and HAM-A, HAM-D, PASS, PDSS, CERQ (18 items) in the PD group. Relationships between the questionnaire measures and average brain activation were examined for the brain regions (dlPFC, dmPFC, and parietal cortex) that differed between PD and HC groups and were associated with explicit and implicit cognitive reappraisal [12, 23]. Considering the amygdala is the core neural substrate of emotion processing, involved in the automatic processing of unpleasant stimuli (especially the perception of anger and fear expressions) and the implicit cognitive reappraisal [23, 46], beta estimates were extracted from the amygdala to investigate the correlation with questionnaire measures.

\section{Results}

\section{Demographics and clinical features}

Table 1 summarizes the demographics and clinical features of PD and HC groups. No remarkable differences were found between the PD and HC groups in sex, age, and years of education. One-way ANOVA demonstrated a major effect that was significant of group on HAM-A and HAM-D $(p<0.001)$. In the CERQ scale, scores relating to catastrophizing and rumination were considerably greater in the PD group than in the HC group, and scores relating to positive reappraisal and putting into perspective were lower in the PD group than in the HC group.

\section{Behavioral task effects}

Results of the valence ratings are presented in Fig. 2. The ANOVA revealed a significant main effect condition $\left(F_{1,49}=38.75, \quad p<0.001, \eta^{2}=0.39\right)$, group $\left(F_{1,49}=7.22\right.$, $\left.p=0.010, \eta^{2}=0.14\right)$, and a Condition $\times$ Group interaction $\left(F_{1,49}=22.19, p<0.001, \eta^{2}=0.24\right)$. Post hoc comparisons were performed using simple effect analysis showed significant differences in ratings of the NNEGDESC and NEG-DESC conditions across the HC group $\left(F_{1,24}=28.53, p<0.001\right)$, but there were no significant differences found in the PD group $\left(F_{1,25}=0.38, p=0.544\right)$.

\section{fMRI task effects}

Results of the whole-brain voxel-wise analysis are shown in Table 2 and Fig. 3. We converted any coordinates in 


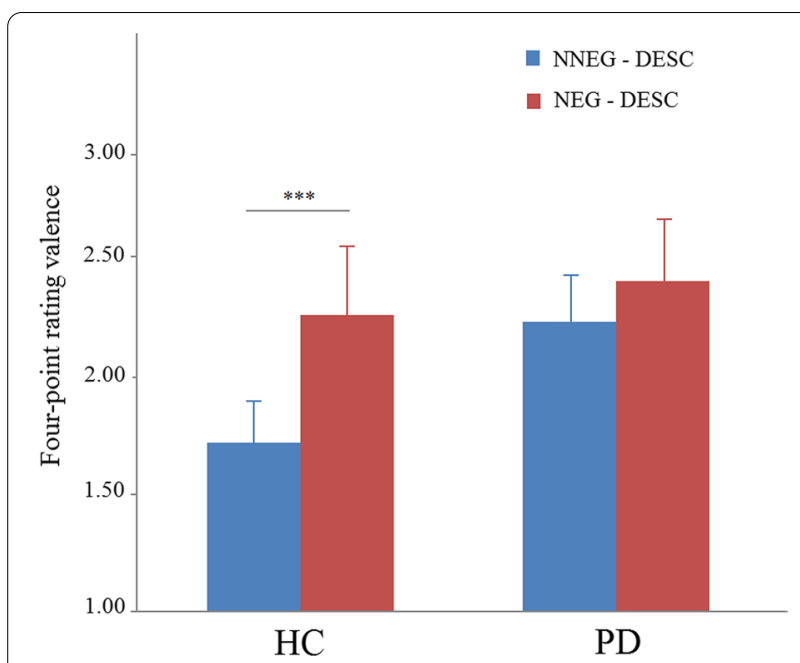

Fig. 2 Negative emotion valence ratings during the task. Asterisks indicate $\mathrm{HC}$ group demonstrated significant difference in valence ratings between the NNEG-DESC and NEG-DESC conditions $\left({ }^{* *} p<0.001\right)$. In PD group, however, no differences in valence ratings between the NNEG-DESC and NEG-DESC conditions were found. HC, healthy control; $P D$, panic disorder

Talairach space reported to MNI space. In the HC group, effects of condition were observed over broad areas including bilateral frontal gyrus, bilateral temporal gyrus, and bilateral parietal gyrus. In contrast, the PD group showed these effects only observed over left frontal and parietal gyrus. Interaction effects were obtained over right parietal cortex (postcentral gyrus, precuneus, and superior parietal lobule, BA 7), and right dIPFC (BA 9) extending to dmPFC (BA 8). Two samples t-test analyses showed that the PD group exhibited less activation (blue color in Fig. 3) in the right dIPFC and right dmPFC in the NNEG-DESC vs NEG-DESC condition, compared to HC. The PD group also exhibited more activation (orange color in Fig. 3) in right parietal cortex during the NNEGDESC condition compared to HC, but not in the NEGDESC condition.

ROI analyses of emotion-related responses in the NNEG-DESC and NEG-DESC conditions in the PD and $\mathrm{HC}$ group were conducted to explore the influences of implicit cognitive reappraisal on the amygdala. As shown in Fig. 4, there were no significant differences as a function of Group $\left(F_{1,49}=2.56, p>0.05, \eta^{2}=0.05\right)$ or Condition $\left(F_{1,49}=1.12, p>0.05, \eta^{2}=0.05\right.$ and while the group $\times$ condition interaction was marginal significance in right amygdala $\left(F_{1,49}=3.6, p=0.06\right.$ corrected, $\eta^{2}=0.07$ ). Simple effects analysis indicated a significant difference between the two conditions in the HC group $\left(t_{24}=2.04, p<0.05\right)$, but not in the PD group $\left(t_{25}=0.15\right.$, $p>0.05)$.

Table 2 Areas of significant decrease and increase in BOLD response in patients and controls during implicit cognitive reappraisal (NNEG-DESC versus NEG-DESC)

\begin{tabular}{|c|c|c|c|c|c|c|c|c|}
\hline & \multirow[t]{2}{*}{ Brain regions } & \multirow[t]{2}{*}{ BA } & \multirow[t]{2}{*}{ Side } & \multirow{2}{*}{$\begin{array}{l}\text { Cluster size } \\
\text { (voxels) }\end{array}$} & \multicolumn{3}{|c|}{ MNI coordinates } & \multirow[t]{2}{*}{ T-value } \\
\hline & & & & & $\mathbf{x}$ & y & $\mathbf{z}$ & \\
\hline \multirow[t]{2}{*}{ PD group: NNEG-DESC > NEG-DESC } & Precentral Gyrus/Postcentral Gyrus & $4 / 6$ & L & 1876 & -40 & -20 & 58 & 6.04 \\
\hline & Cerebellum & - & $\mathrm{R}$ & 691 & 18 & -86 & -40 & 4.63 \\
\hline \multirow[t]{8}{*}{ HC group: NNEG-DESC > NEG-DESC } & $\begin{array}{l}\text { Postcentral Gyrus/Precentral Gyrus/Inferior } \\
\text { Parietal Loule }\end{array}$ & $3 / 7 / 40$ & $\mathrm{R}$ & 1371 & 47 & -29 & 58 & -5.92 \\
\hline & $\begin{array}{l}\text { Dorsolateral prefrontal cortex/Dorsomedial } \\
\text { prefrontal cortex }\end{array}$ & $6 / 8 / 9$ & $L$ & 771 & -43 & 20 & 41 & 5.8 \\
\hline & Lateral Orbitofrontal Cortex & $10 / 11$ & L & 523 & -40 & 43 & -22 & 4.56 \\
\hline & Cerebellum & - & $\mathrm{R}$ & 432 & 15 & -80 & -37 & 5.21 \\
\hline & $\begin{array}{l}\text { Angular Gyrus/Precuneus/Inferior Parietal } \\
\text { Lobule }\end{array}$ & $19 / 39$ & $\mathrm{R}$ & 409 & 40 & -74 & 48 & 4.90 \\
\hline & Middle Temporal Gyrus/Inferior Temporal Gyrus & $20 / 21$ & $\mathrm{R}$ & 399 & 62 & -44 & -13 & 4.34 \\
\hline & $\begin{array}{l}\text { Postcentral Gyrus/Precuneus/Superior parietal } \\
\text { lobule }\end{array}$ & 7 & R & 366 & 37 & 55 & -23 & 4.81 \\
\hline & $\begin{array}{l}\text { Angular Gyrus/Middle Temporal Gyrus/Precu- } \\
\text { neus }\end{array}$ & $19 / 39$ & L & 271 & -40 & -65 & 44 & 4.64 \\
\hline \multirow{3}{*}{$\begin{array}{l}\text { Interaction effects: HC(NNEG- } \\
\text { DESC > NEG-DESC) > PD (NNEG- } \\
\text { DESC > NEG-DESC) }\end{array}$} & $\begin{array}{l}\text { Postcentral Gyrus/precuneus/Superior parietal } \\
\text { lobule }\end{array}$ & 7 & R & 295 & 20 & -51 & 66 & 4.33 \\
\hline & Dorsolateral prefrontal cortex ext & $6 / 9$ & $\mathrm{R}$ & 230 & 11 & 27 & 60 & -3.82 \\
\hline & Dorsomedial prefrontal cortex & 8 & R & & 9 & 19 & 51 & -3.22 \\
\hline
\end{tabular}

$P D$ panic disorder, $H C$ healthy control, ext extending into, $B A$ Brodmann area, $L$ left, $R$ right

$\mathrm{xyz}=\mathrm{MNI}$ coordinates of the peak active voxel 


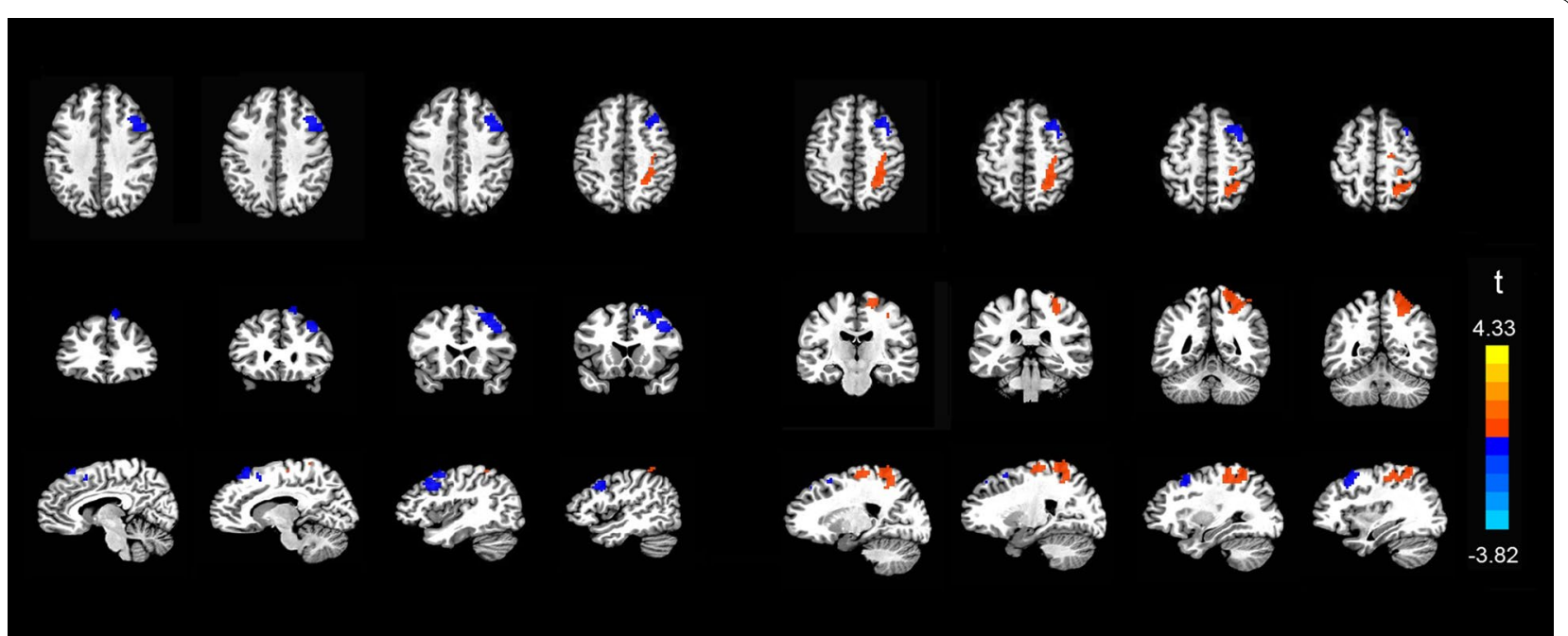

Fig. 3 Whole-brain analysis. Group $\times$ task interaction shows that the PD group, compared to HC, exhibits less activation (blue color) in the right dIPFC and right dmPFC and more activation (orange color) in right parietal cortex during implicit cognitive reappraisal (NNEG-DESC vs NEG-DESC). Activations were thresholded at $p<0.05$, corrected. PD, panic disorder; $\mathrm{HC}$, healthy control

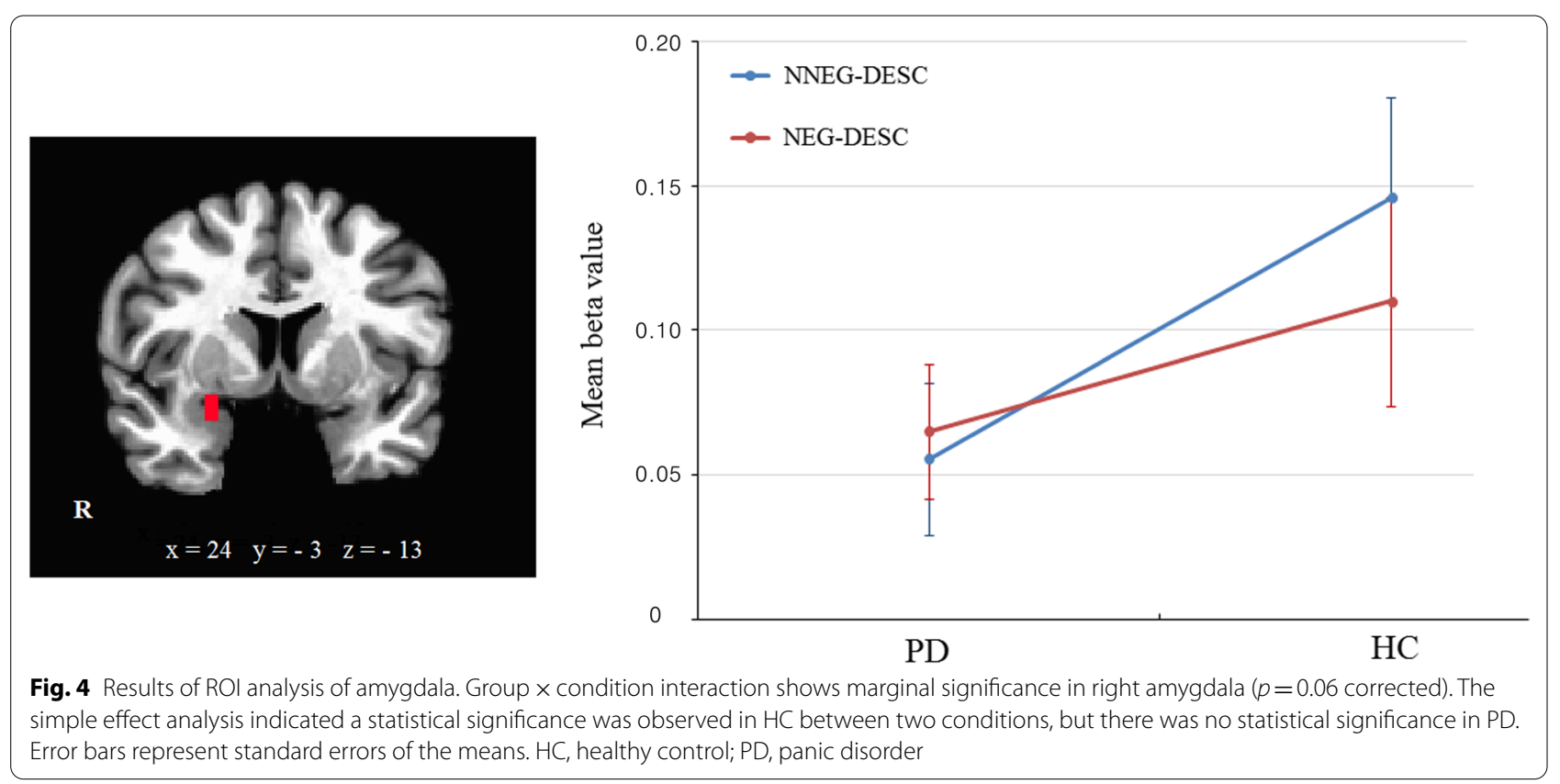

\section{Brain-behavior relationships}

We found no evidence that self-reported valence ratings were related to beta estimates drawn from the dlPFC, dmPFC, parietal cortex, and amygdale during NNEGDESC, NEG-DESC, and NNEG-DESC vs NEG-DESC within the PD and HC groups ( $p>0.05)$.

Relationships between symptom severity obtained from questionnaires and average brain activation during cognitive reappraisal (NNEG-DESC vs NEG-DESC) were examined for the two brain regions (right dlPFC/dmPFC and right parietal cortex) in the PD group. Owing to peak activation of dlPFC extending to dmPFC, we selected dIPFC to analyze as a whole. The results showed that those with greater higher score of HAM-A showed relatively less activation in right dIPFC/dmPFC during NNEG-DESC vs NEG-DESC ( $r=-0.44, p=0.026)$ (Fig. 5A). Similarly, PD patients with higher score of PDSS showed relatively less activation in right dIPFC/dmPFC during NNEG-DESC vs 


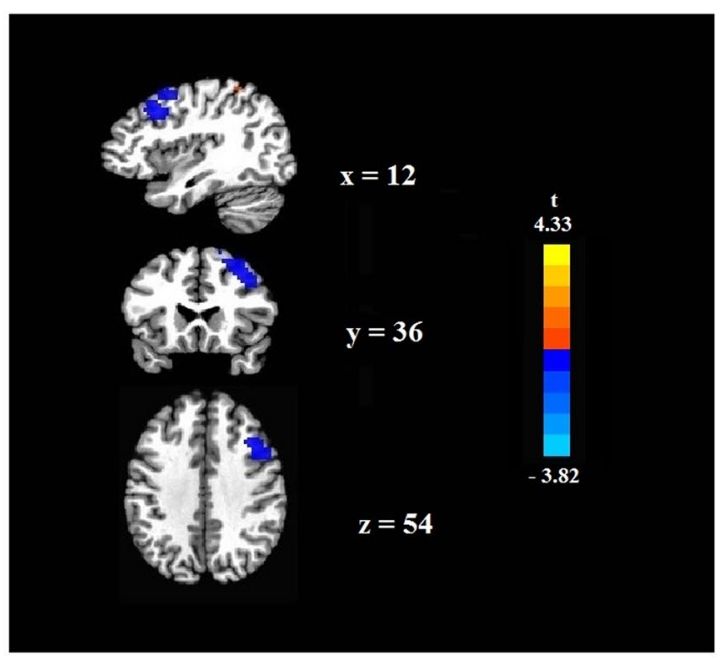

A
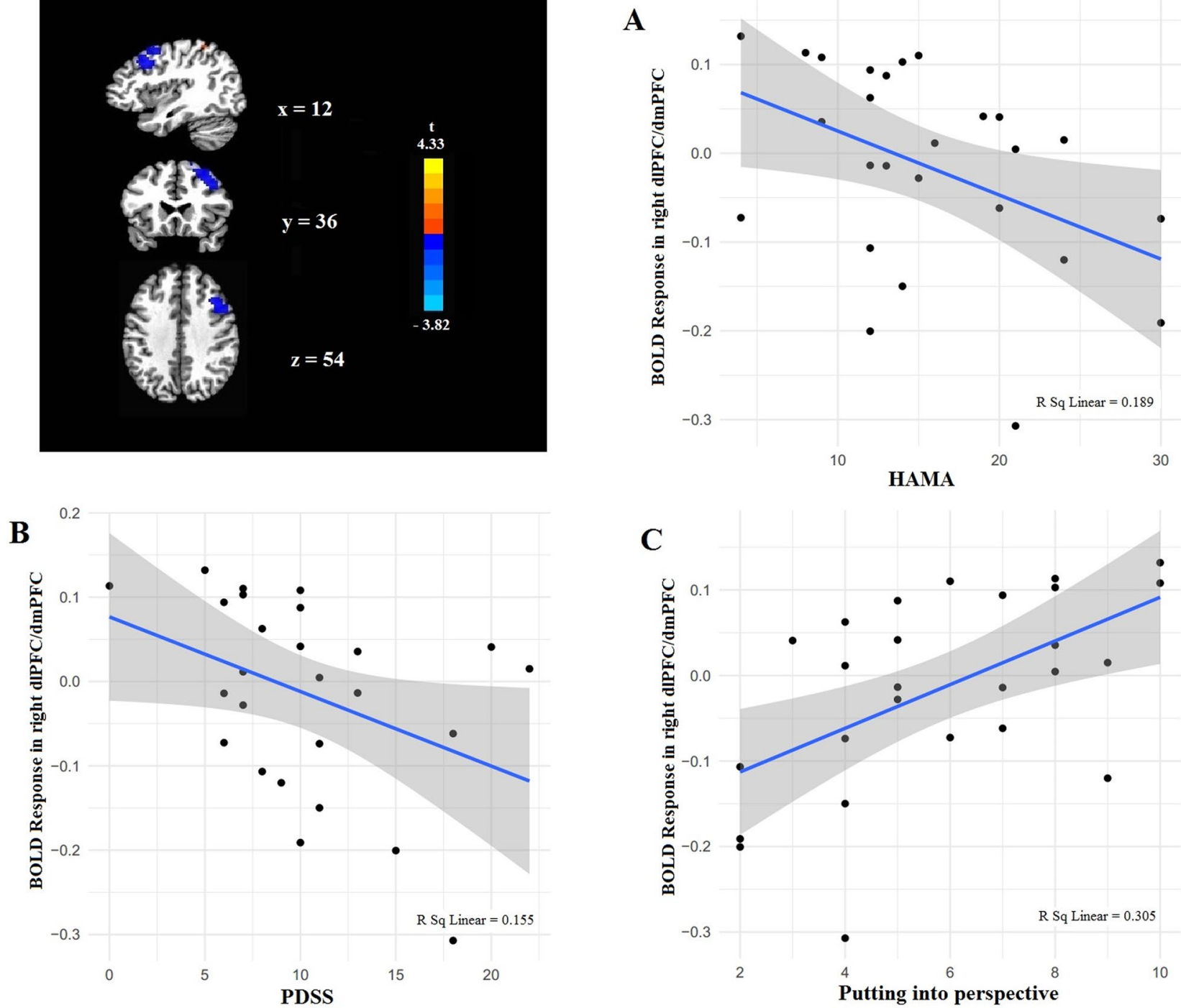

Fig. 5 Relationships between significant clusters of activation during implicit cognitive reappraisal and symptom severity in PD. A Greater higher score of HAM-A showed relatively less activation in right dIPFC/dmPFC. B Greater higher score of PDSS showed relatively less activation in right dIPFC/dmPFC. C Positive relationship between the score of putting into perspective in CERQ and activation in right dIPFC/dmPFC. PD, panic disorder; HAM-A, Hamilton Anxiety Rating Scale; PDSS, Panic Disorder Severity Scale; CERQ, Cognitive Emotion Regulation Questionnaire; dorsolateral prefrontal cortex,dIPFC; dorsomedial prefrontal cortex, dmPFC

NEG-DESC $(r=-0.39, p=0.046)$ (Fig. 5B). We additionally found a positive relationship between the score of putting into perspective in CERQ and activation in right dlPFC/ dmPFC during NNEG-DESC vs NEG-DESC $(r=0.55$, $p=0.003$ ) (Fig. 5C). We found no relationship between brain activation and any other variables $(p>0.05)$.

\section{Discussion}

The present study explored how PD affects brain functioning in an implicit reappraisal strategy when applied to negative picture processing. We found that the neutral/positive description decreased negative valence ratings compared to negative description when presented to healthy subjects. In contrast, there was no significant effect of prior descriptions on valence ratings for PD participants. Besides, we found interactive effects of group and condition in the right amygdala but was not affected during implicit reappraisal in the PD group, suggesting that this strategy was not an effective neural modulator of negative emotions in PD. Moreover, whole-brain results showed that $\mathrm{PD}$ patients exhibited less activation in the right dlPFC and right dmPFC 
and greater activation in parietal cortex during the NNEG-DESC vs NEG-DESC conditions, compared to $\mathrm{HC}$. These findings appear to be in support of a damaged automatic top-down regulation due to prefrontal dysfunction which could not decrease responses to negative emotion in the amygdala. Finally, activation of dIPFC and dmPFC was negatively correlated with anxiety and panic severity in $\mathrm{PD}$, suggesting that the failure to recruit prefrontal cortex in implicit cognitive reappraisal might be associated with the severity of anxiety and panic.

Implicit emotion regulation is considered when the subject has no subjective awareness or effort to influence the emotion regulation process but eventually attenuates the emotional response [22, 47]. In our task, subjects received a short description of the upcoming pictures and then simply watched the pictures. With this approach, subjects were given descriptions that could unconsciously influence the meaning of the forthcoming pictures, rather than leaving them to generate their own reinterpretations. In passive viewing of negative pictures, subjects simply waiting and watching, and not being instructed to moderate their emotional responses. The cognitive regulatory processes are activated and occur outside of consciousness. During this regulatory process, subjects may have an implicit goal, provoked and sustained by previous descriptions, to regulate their emotional feelings upon viewing the negative pictures. Therefore, this cognitive emotion regulation processes were effortless, uninstructed, and proceeds without awareness.

Implicit emotion regulation occurs only under the influence of certain external stimuli, which are essential for implicit emotion regulation to occur [48]. In our task, the neutral/positive interpretation preceding the negative picture induces a specific emotion in the subject, and the matching of that specific emotion with the negative picture serves as an external stimulus to elicit implicit regulatory processes. Using implicit cognitive reappraisal task with ERP, Mocaiber et al. showed that if subjects passively viewed emotional pictures produced smaller LPP amplitudes than non-emotional pictures or no significant difference was observed, it was inferred that subjects showed implicit emotion regulation [49]. Similarly, in our earlier study, this implicit cognitive reappraisal paradigm was used with fMRI to successfully explore the neural mechanisms of implicit emotion regulation in healthy individuals [23]. The process of emotion regulation described in the above study is implicit: individuals are unaware of the regulation of emotional control elicited by the stimuli on their behavior, and the regulatory process takes place largely outside of conscious awareness. These studies allow us to more reliably explore the neural basis of implicit emotion dysregulation of PD in the current study.

Reinterpretation and distancing are two main tactics studied under reappraisal [29]. It would be fruitful to discuss the differences and commonalities between reinterpretation and distancing. The distinction of these two strategies is that reinterpretation concentrates more on altering the meaning or content of the stimulus, whereas distancing emphasizes more on shifting the perspective of considering the stimulus. Both tactics are effective and favorable over other emotion regulation strategies in some contexts [29], while the two strategies were studied separately in the single study revealing that reinterpretation appears to activate more ventrolateral prefrontal cortex, whereas alienation is related to activation of parietal cortex [13]. Ochsner et al. [50] directly compared the neural basis of reinterpretation and distancing, finding increased activation in the lateral prefrontal cortex, temporal, parietal, and occipital cortex in subjects using reinterpretation and greater activation in the cingulate gyrus and parietal cortex in the group using distancing. A further between-subjects study demonstrated increased activation in brain regions including orbitofrontal cortex, frontal cortex, insula, supplementary motor areas, parietal cortex, and temporal cortex in reinterpretation compared with distancing, which more strongly enhanced activation in the parietal cortex [51]. Thus, these prior findings suggest that the prefrontal cortex involving lateral prefrontal cortex, supplementary motor areas, and orbitofrontal cortex, temporal cortex and parietal cortex were recruited in reinterpretation, while the parietal cortex was associated with distancing. Notably, distancing may be a particularly promising strategy out of the two and that the benefits of distancing motivate further investigation of the tactic [29].

According to Ochsner's multi-level framework of implicit emotion regulation [52], implicit-controlled emotion regulation is marked with an implicit emotional regulation goal and the involvement of active control processes. In the paradigm used in our study, reinterpretation was considered as an active control that is involved in active cognitive control processes initiated by implicit goals, which is consistent with Ochsner's implicit-control emotion regulation framework. Thus, we believe that reinterpretation may be appropriate for implicit condition.

In earlier work, we explored the neural bases of implicit emotion regulation in $\mathrm{HC}$ by using the implicit interpretation paradigm and demonstrated that implicit interpretation processes could recruit prefrontal areas involving prefrontal cortex involving the dIPFC and dmPFC, and parietal cortex [23]. In the current study, PD patients exhibited less activation in the 
dlPFC and dmPFC compared to HC, which coincides with brain regions involved in implicit cognitive reappraisal in $\mathrm{HC}$ and also overlaps and differs from the brain regions involved in the explicit reinterpretation. Thus, increased activation in prefrontal areas generally associated with explicit forms of reinterpretation may be also partly engaged in implicit reinterpretation.

The lack of effect of valence ratings between the NNEG-DESC and NEG-DESC conditions in the PD group suggests that when viewing the negative images proceeded by non-negative descriptions, PD subjects were not able to use implicit reappraisal to reduce subjective negative experience. Significantly, this result contrasts with Ball et al's report that PD individuals can successfully regulate emotions by use of explicit cognitive reappraisal strategies that require participants to deliberately transform aversive stimuli into less negative interpretation $[9,12]$. As emotion regulation impairment in PD could operate at an unconscious level of cognitive appraisal $[18,28]$, such an explicit reappraisal paradigm may not sufficiently reveal the pathogenesis of PD.

The PD group also exhibited less activation in the right dIPFC and right dmPFC relative to HC during NNEGDESC condition vs NEG-DESC condition. DIPFC is regarded as the core node of the regulatory network and is associated with motor suppression, working memory, reasoning, and complex cognition [12, 53]. Moreover, dlPFC has an important role in top-down cognitive control $[23,54,55]$ and explicit regulation [13, 52]. Previous studies $[9,56-58]$ have shown hypoactivation in the dlPFC in tasks of explicit reappraisal in participant samples with anxiety and mood disorders. In a recent study, activation in dlPFC was associated with automatic cognitive top-down control using an implicit reappraisal paradigm to explore mechanisms of implicit reappraisal in healthy participants [23]. Therefore, it is possible that the impaired dIPFC in PD patients results in insufficient engagement of automatic attentional and inhibitory control, biasing patients' attitudes toward aversive stimuli.

PD patients showed active clusters extending to dmPFC in dIPFC, but not in HC, when comparing NNEG-DESC and NEG-DESC conditions. The dmPFC is associated with elaborating the affective meaning of stimuli and monitoring emotional experiences [12] and is also related to implicit cognitive reappraisal [7]. Numerous studies from cognitive reappraisal suggest that healthy subjects could engage dmPFC to enable successful emotion regulation $[6,53]$. Findings from our study, therefore, suggest that emotion regulation alterations in PD may be partially a consequence of ineffective management of monitoring and reflecting upon the implications of altered emotional stimulation in implicit cognitive reappraisal.
Collectively, this results in a decreased capacity to automatically down-regulate negative responsiveness.

PD patients also showed involvement of the increased activation in right parietal cortex compared to $\mathrm{HC}$ in contrasting the two conditions. This cerebral region is part of the prefrontal-parietal network [7] associated with sensory information, in which response-relevant information from the top-down promotes flexible, goal-directed behavior [59]. Studies in healthy controls and individuals with pathological health anxiety have revealed that a hyper-activated parietal cortex is implicated in implicit emotional processing $[60,61]$. As the prefrontal cortex and parietal cortex are the two important parts of frontoparietal network involving in implicit cognitive reappraisal [23], the increased activity of parietal cortex may be a compensatory mechanism for reduced recruitment of dlPFC and dmPFC during implicit reappraisal. This tentative interpretation suggests that dysfunction in the frontoparietal network responsible for the implicit cognitive control of negative emotions may be a characteristic feature of PD and that the hyperactivation may appear in parietal cortex as a consequence of, or to compensate for, impaired emotion regulation.

We further observed a Group $\times$ Condition interaction in the right amygdala for the $\mathrm{HC}$ group, but not the PD group, during implicit reappraisal. According to a prominent neurobiological model of emotion regulation, threat processing is related to signaling in limbic areas of the brain, such as the amygdala, a critical area associated with the sensing and encoding of adverse stimuli, and the successful down-regulation of this response is considered to be implicated in an increase in cognitive control of prefrontal areas $[13,62]$. A recent review showed that the core concept for pathophysiology in panic disorder might be linked with disturbances in the frontal-limbic network [63]. Therefore, this result indicates that prefrontal lobes of PD patients may not sufficiently engage unconscious top-down control to decrease the activity in amygdala.

Using CERQ, we found that PD patients use less positive reappraisal and putting into perspective but more catastrophic strategies such as catastrophizing and rumination. Rumination is regarded as maladaptive and has been related to greater levels of self-reported anxiety symptoms [64], while catastrophic cognition is closely related to triggering and maintaining panic [17]. When perceiving negative images, individuals with $\mathrm{PD}$ give priority to self-reflection and think more about the effects of negative events, while simultaneously adapting the catastrophizing cognitive model to emphasize the negative components of emotional events to exaggerate perceptions of threat. This chain of events consequently enhances the intensity of emotional response to stimuli and triggers panic attacks and pathological anxiety [65]. 
Hence, this cognitive bias compels patients to implicitly choose to focus on and exaggerate threatening components when viewing aversive images, making it difficult to gather cognitive resources to adjust their emotional state to align with previous neutral/positive descriptions.

Interestingly, we observed inverse correlations between anxiety severity and dlPFC/dmPFC activation in the PD group, consistent with a prior report that anxiety severity and functional impairment are inversely associated with prefrontal activation [8]. Panic severity is negatively correlated with dIPFC/dmPFC activation during implicit reappraisal, in which the severity of panic symptoms over the last month was correlated with weaker prefrontal activity. Such findings suggest that the failure to engage the prefrontal cortex during implicit cognitive reappraisal might be associated with the severity of anxiety and panic symptoms. Furthermore, the positive relationship between putting into perspective score and activation in right prefrontal cortex was found during implicit cognitive reappraisal. The putting into perspective is the tendency to reduce the significance of a circumstance relative to other experiences [66] and is associated with the improvement of anxiety symptoms [64], implicating a promising quantitative indicator for PD treatment.

There are several limitations to this study. First, previous studies have shown regions of the prefrontal cortex associated with implicit reappraisal that could be overlapping in explicit reappraisal to modulate amygdala activation [23]. Although our results showed that dlPFC, dmPFC, and parietal cortex in PD are engaged during implicit reappraisal, these brain regions are also related to explicit reappraisal to some extent. Moreover, the PD-related changes in brain function between unconscious and conscious reappraisal were not directly compared. Further studies should elucidate the differences and commonalities of both emotion regulation strategies. Second, some patients with PD presented with co-morbid major depression, reducing our ability to classify neural engagement specific to PD. However, the high rate of co-morbidity in anxiety disorders [39] including individuals with depressive disorder or other anxiety disorders, may increase the generalizability of those with PD in community and clinical settings. Third, in this experiment we have attempted to focus our examination on differences in brain activation between PD and $\mathrm{HC}$ during implicit cognitive reappraisal. This study is not longitudinal in design. Longitudinal studies of the same subjects should be performed in future to further refine establish causality and neural activity changes in PD during implicit cognitive reappraisal. Fourth, as the other main reappraisal tactics for emotion regulation compared to reinterpretation, psychological distancing may be particular promising in psychopathological disorders [29], thus future studies could utilize an implicit psychological distancing strategy to more clearly differentiate the processes that are impaired in PD. Fifth, since the current study was conducted on individuals of Chinese Asians, another limitation is that these findings lack representativeness to all individuals and therefore should be generalized only to the appropriate race/ demographic. Finally, there was a lack of neutral images in our experimental task that make it hard to separate the attenuation caused by non-negative description from the enhancement caused by negative description. Further studies should attempt to disentangle the two in order to clarify the neural mechanisms involved in the implicit cognitive reappraisal of PD.

\section{Conclusion}

Patients with PD showed a different pattern of brain activation from $\mathrm{HC}$ when performing an implicit cognitive reappraisal task. Specifically, patients were not able to recruit some of the prefrontal regions (i.e., dlPFC and dmPFC) to modulate emotional responses in the amygdala, suggesting that emotional dysregulation in PD is likely the result of compromised top-down, automatic regulation of negative emotions. These results provide a valuable target for future research evaluating therapeutic interventions for PD that rely on implicit reappraisal (e.g., cognitive behavioral therapy) $[67,68]$ or neuromodulatory interventions (e.g., transcranial magnetic stimulation) [69]. A negative relationship between severity of anxiety and panic and activation of right dlPFC and $\mathrm{dmPFC}$ in the present study demonstrate that the failure to engage prefrontal cortex during implicit cognitive reappraisal might be associated with the severity of anxiety and panic symptoms. This study sheds new light on the neural dysfunction underlying PD during emotion regulation, highlighting the important role of functional changes in dIPFC and dmPFC that could be useful in understanding the neuropathological mechanisms underscoring PD and how to treat it.

\footnotetext{
Abbreviations

PD: Panic disorder; HC: Healthy controls; dIPFC: Dorsolateral prefrontal cortex; dmPFC: Dorsomedial prefrontal cortex; ERPs: Event-related potentials; LPP: Late positive potential; DSM-5: Diagnostic and statistical manual of mental disorders, 5th Edition; HAM-A: Hamilton Anxiety Rating Scale; HAM-D: Hamilton Depression Rating Scale; PASS: Panic-Associated Symptom Scale; PDSS: Panic Disorder Severity Scale; CERQ: Cognitive Emotion Regulation Questionnaire; NEG-DESC: Negative descriptions preceding negative pictures condition; NNEG-DESC: Non-negative descriptions preceding negative pictures condition; ANOVA: Analysis of variance; BOLD: Blood oxygenation level-dependent; FOV: Field of view; AFNI: Analysis of Functional Neurolmages; ROI: Regions-of-interest.
} 


\section{Acknowledgements \\ Not applicable.}

\section{Authors' contributions}

Wrote the paper: H-YW. Data collection and analysis: H-YW, G-QX, M-FN, C-HZ, $X-L L, Y C, X-P S$, and B-WZ. Conceived and designed the study: B-WZ. All authors have read and approved the final manuscript.

\section{Funding}

This work was supported by the National Natural Science Foundation of China (No. 81871080 and 81401486), the Natural Science Foundation of Liaoning Province of China (No. 20170540276 and 2019-MS-099), and the Medicine and Health Science Technology Development Program of Shandong Province (202003070713).

\section{Availability of data and materials}

The datasets used and/or analyzed during the current study are available from the corresponding author on reasonable request.

\section{Declarations}

\section{Ethics approval and consent to participate}

The study protocol was approved by the First Affiliated Hospital of Dalian Medical University (KY2014-30) was carried out in agreement with the provisions of the Declaration of Helsinki. Written informed consent was obtained from all patients.

\section{Consent for publication}

Not applicable.

\section{Competing interests}

The authors declare that they have no competing interests.

\section{Author details}

${ }^{1}$ Department of Neurology, Jining No. 1 People's Hospital, Jining 272000, China. ${ }^{2}$ Department of Neurology and Psychiatry, The First Affiliated Hospital of Dalian Medical University, No.222, Zhongshan Road, Dalian 116011, Liaoning Province, China. ${ }^{3}$ Department of Psychology, Dalian Medical University, Dalian 116044, China. ${ }^{4}$ Department of Radiology, The First Affiliated Hospital of Dalian Medical University, Dalian 116011, China. ${ }^{5}$ Department of Geriatric Medicine, Huizhou Third People's Hospital, Guangzhou Medical University, Huizhou 516000, China. ${ }^{6}$ Department of Intensive Care Unit, Jining No. 1 People's Hospital, Jining 272000, China.

Received: 11 January 2021 Accepted: 28 June 2021

Published online: 13 July 2021

\section{References}

1. McRae K, Gross JJ. Emotion regulation. Emotion. 2020;20(1):1-9.

2. Ding N, Yang J, Liu Y, Yuan J. Paying less but harvesting more: the effect of unconscious acceptance in regulating frustrating emotion. Sci China Life Sci. 2015;58(8):799-809.

3. Gross JJ. Emotion Regulation: current status and future prospects. Psychol Inq. 2015;26(1):1-26.

4. Berking $M$, Wupperman P. Emotion regulation and mental health: recent findings, current challenges, and future directions. Curr Opin Psychiatry. 2012:25(2):128-34.

5. Association AP. Diagnostic and statistical manual of mental disorders (DSM- $5^{\circledR}$ ). Washington: American Psychiatric Association Publishing 2013

6. Zilverstand A, Parvaz MA, Goldstein RZ. Neuroimaging cognitive reappraisal in clinical populations to define neural targets for enhancing emotion regulation. A systematic review. Neuroimage. 2017;151:105-16.

7. Wang HY, Zhang XX, Si CP, Xu Y, Liu Q, Bian HT, et al. Prefrontoparietal dysfunction during emotion regulation in anxiety disorder: a meta-analysis of functional magnetic resonance imaging studies. Neuropsychiatr Dis Treat. 2018;14:1183-98.
8. Reinecke A, Filippini N, Berna C, Western DG, Hanson B, Cooper MJ, et al. Effective emotion regulation strategies improve $\mathrm{AMRI}$ and ECG markers of psychopathology in panic disorder: implications for psychological treatment action. Transl Psychiatry. 2015;5(11):e673.

9. Ball TM, Ramsawh HJ, Campbell-Sills L, Paulus MP, Stein MB. Prefrontal dysfunction during emotion regulation in generalized anxiety and panic disorders. Psychol Med. 2013;43(7):1475-86.

10. Zhang BW, Xu J, Chang Y, Wang H, Yao H, Tang D. Impaired cognitive reappraisal in panic disorder revealed by the late positive potential. Neuroreport. 2016;27(2):99-103.

11. Gross JJ. Antecedent- and response-focused emotion regulation: divergent consequences for experience, expression, and physiology. J Pers Soc Psychol. 1998;74(1):224-37.

12. Buhle JT, Silvers JA, Wager TD, Lopez R, Onyemekwu C, Kober H, et al. Cognitive reappraisal of emotion: a meta-analysis of human neuroimaging studies. Cereb Cortex. 2014;24(11):2981-90.

13. Ochsner KN, Silvers JA, Buhle JT. Functional imaging studies of emotion regulation: a synthetic review and evolving model of the cognitive control of emotion. Ann N Y Acad Sci. 2012;1251:E1-24.

14. Teachman BA, Smith-Janik SB, Saporito J. Information processing biases and panic disorder: relationships among cognitive and symptom measures. Behav Res Ther. 2007:45(8):1791-811.

15. McNally RJ. Anxiety sensitivity and panic disorder. Biol Psychiatry. 2002;52(10):938-46.

16. Clark DM. A cognitive approach to panic. Behav Res Ther. 1986;24(4):461-70.

17. Pilecki B, Arentoft A, McKay D. An evidence-based causal model of panic disorder. J Anxiety Disord. 2011;25(3):381-8.

18. Zhang $B$, Jing $X$, Wang $H$, Yao $H$, Zhang $L$, Liu X. Cognitive emotion regulation strategies in subjects with panic disorder. Chin J Behav Med Brain Sci. 2014:23(6):484-6.

19. Salkovskis PM, Clark DM, Hackmann A, Wells A, Gelder MG. An experimental investigation of the role of safety-seeking behaviours in the maintenance of panic disorder with agoraphobia. Behav Res Ther. 1999:37(6):559-74.

20. Baker R, Holloway J, Thomas PW, Thomas S, Owens M. Emotional processing and panic. Behav Res Ther. 2004;42(11):1271-87.

21. Breuninger C, Sláma DM, Krämer M, Schmitz J, Tuschen-Caffier B. Psychophysiological reactivity, interoception and emotion regulation in patients with agoraphobia during virtual reality anxiety induction. Cogn Ther Res. 2017;41(2):193-205.

22. Yuan J, Ding N, Liu Y, Yang J. Unconscious emotion regulation: Nonconscious reappraisal decreases emotion-related physiological reactivity during frustration. Cogn Emot. 2015;29(6):1042-53.

23. Wang HY, Xu GQ, Ni MF, Zhang CH, Sun XP, Chang Y, et al. Neural mechanisms of implicit cognitive reappraisal: preceding descriptions alter emotional response to unpleasant images. Neuroscience. 2017;347:65-75.

24. Roy-Byrne PP, Craske MG, Stein MB. Panic disorder. Lancet. 2006;368(9540):1023-32.

25. Etkin A, Prater KE, Hoeft F, Menon V, Schatzberg AF. Failure of anterior cingulate activation and connectivity with the amygdala during implicit regulation of emotional processing in generalized anxiety disorder. Am J Psychiatry. 2010;167(5):545-54.

26. Chang $Y, X u$ J, Pang $X$, Sun $Y$, Zheng $Y$, Liu Y. Mismatch negativity indices of enhanced preattentive automatic processing in panic disorder as measured by a multi-feature paradigm. Biol Psychol. 2015;105:77-82.

27. Tang D, Xu J, Chang Y, Zheng Y, Shi N, Pang X, et al. Visual mismatch negativity in the detection of facial emotions in patients with panic disorder. Neuroreport. 2013;24(5):207-11.

28. Li XL, Wang HY. Unconscious cognitive dysfunction in emotion dysregulation and psychopathology of panic disorder: evidence from the late positive potential. Neuroreport. 2018;29(1):6-7.

29. Powers JP, LaBar KS. Regulating emotion through distancing: a taxonomy, neurocognitive model, and supporting meta-analysis. Neurosci Biobehav Rev. 2019:96:155-73.

30. Foti $D$, Hajcak G. Deconstructing reappraisal: descriptions preceding arousing pictures modulate the subsequent neural response. J Cogn Neurosci. 2008;20(6):977-88 
31. Burklund LJ, Creswell JD, Irwin MR, Lieberman MD. The common and distinct neural bases of affect labeling and reappraisal in healthy adults. Front Psychol. 2014;5:221.

32. Meyer ML, Berkman ET, Karremans JC, Lieberman MD. Incidental regulation of attraction: the neural basis of the derogation of attractive alternatives in romantic relationships. Cogn Emot. 2011;25(3):490-505.

33. Kent JM, Rauch SL. Neurocircuitry of anxiety disorders. Curr Psychiatry Rep. 2003;5(4):266-73.

34. Hamilton M. The assessment of anxiety states by rating. Br J Med Psychol. 1959;32(1):50-5.

35. Hamilton M. A rating scale for depression. J Neurol Neurosurg Psychiatry. 1960;23(1):56-62.

36. Argyle N, Deltito J, Allerup P, Maier W, Albus M, Nutzinger D, et al. The panic-associated symptom scale: measuring the severity of panic disorder. Acta Psychiatr Scand. 1991;83(1):20-6.

37. Shear MK, Brown TA, Barlow DH, Money R, Sholomskas DE, Woods SW, et al. Multicenter collaborative panic disorder severity scale. Am J Psychiatry. 1997;154(11):1571-5.

38. Garnefski N, Kraaij V. Cognitive emotion regulation questionnairedevelopment of a short 18-item version (CERQ-short). Pers Individ Dif. 2006;41(6):1045-53.

39. Kessler RC, Chiu WT, Demler O, Merikangas KR, Walters EE. Prevalence, severity, and comorbidity of 12-month DSM-IV disorders in the National Comorbidity Survey Replication. Arch Gen Psychiatry. 2005;62(6):617-27.

40. He YL, Zeng QZ, Wei J, Shi YX, Zhang HY, Wu WY, et al. Reliability and validity of the Chinese version of panic disorder severity scale and panicassociated symptom scale. Chin J Psychiatry. 2013;46(04):217-21.

41. Zhu X, Luo F, Yao S, Auerbach RP, Abela JRZ. The reliability and validity of the Chinese version of the cognitive emotion regulation questionnaire (CERQ-C). Chin J Clin Psychol. 2007;15(2):121-4.

42. Lang P, Bradley M, Cuthbert BN. International Affective Picture System (IAPS): Affective ratings of pictures and instruction manual. Technical Report A-8. Gainesville: University of Florida; 2008.

43. Cox RW. AFNI: software for analysis and visualization of functional magnetic resonance neuroimages. Comput Biomed Res. 1996;29(3):162-73.

44. Cox RW, Chen G, Glen DR, Reynolds RC, Taylor PA. fMRI clustering and false-positive rates. Proc Natl Acad Sci USA. 2017;114(17):E3370-1.

45. Taschereau-Dumouchel V, Cortese A, Chiba T, Knotts JD, Kawato M, Lau H. Towards an unconscious neural reinforcement intervention for common fears. Proc Natl Acad Sci USA. 2018;115(13):3470-5.

46. Burnett S, Sebastian C, Cohen Kadosh K, Blakemore SJ. The social brain in adolescence: evidence from functional magnetic resonance imaging and behavioural studies. Neurosci Biobehav Rev. 2011;35(8):1654-64.

47. Mauss IB, Bunge SA, Gross JJ. Automatic emotion regulation. Soc Personal Psychol Compass. 2007;1(1):146-67.

48. Gyurak A, Gross JJ, Etkin A. Explicit and implicit emotion regulation: a dual-process framework. Cogn Emot. 2011;25(3):400-12.

49. Mocaiber I, Pereira MG, Erthal FS, Machado-Pinheiro W, David IA, Cagy M, et al. Fact or fiction? An event-related potential study of implicit emotion regulation. Neurosci Lett. 2010;476(2):84-8.

50. Ochsner KN, Ray RD, Cooper JC, Robertson ER, Chopra S, Gabrieli JD, et al. For better or for worse: neural systems supporting the cognitive downand up-regulation of negative emotion. Neuroimage. 2004;23(2):483-99.

51. Dörfel D, Lamke JP, Hummel F, Wagner U, Erk S, Walter H. Common and differential neural networks of emotion regulation by detachment, reinterpretation, distraction, and expressive suppression: a comparative fMRI investigation. Neuroimage. 2014;101:298-309.

52. Braunstein LM, Gross JJ, Ochsner KN. Explicit and implicit emotion regulation: a multi-level framework. Soc Cogn Affect Neurosci. 2017:12(10):1545-57.
53. Kohn N, Eickhoff SB, Scheller M, Laird AR, Fox PT, Habel U. Neural network of cognitive emotion regulation-an ALE meta-analysis and MACM analysis. Neuroimage. 2014;87:345-55.

54. Woo YK, Song J, Jiang Y, Cho C, Bong M, Kim SI. Effects of informative and confirmatory feedback on brain activation during negative feedback processing. Front Hum Neurosci. 2015;9:378.

55. Rive MM, van Rooijen G, Veltman DJ, Phillips ML, Schene AH, Ruhé HG Neural correlates of dysfunctional emotion regulation in major depressive disorder. A systematic review of neuroimaging studies. Neurosci Biobehav Rev. 2013;37((10 Pt 2)):2529-53.

56. Warren SL, Zhang Y, Duberg K, Mistry P, Cai W, Qin S, et al. Anxiety and stress alter decision-making dynamics and causal amygdala-dorsolateral prefrontal cortex circuits during emotion regulation in children. Biol Psychiatry. 2020;88(7):576-86.

57. Erk S, Mikschl A, Stier S, Ciaramidaro A, Gapp V, Weber B, et al. Acute and sustained effects of cognitive emotion regulation in major depression. J Neurosci. 2010;30(47):15726-34.

58. Townsend JD, Torrisi SJ, Lieberman MD, Sugar CA, Bookheimer SY, Altshuler LL. Frontal-amygdala connectivity alterations during emotion downregulation in bipolar I disorder. Biol Psychiatry. 2013;73(2):127-35.

59. Dodds CM, Morein-Zamir S, Robbins TW. Dissociating inhibition, attention, and response control in the frontoparietal network using functional magnetic resonance imaging. Cereb Cortex. 2011;21(5):1155-65.

60. Suslow T, Kugel H, Lindner C, Dannlowski U, Egloff B. Brain response to masked and unmasked facial emotions as a function of implicit and explicit personality self-concept of extraversion. Neuroscience. 2017;340:464-76

61. Yan Z, Witthöft M, Bailer J, Diener C, Mier D. Scary symptoms? Functional magnetic resonance imaging evidence for symptom interpretation bias in pathological health anxiety. Eur Arch Psychiatry Clin Neurosci. 2019;269(2):195-207.

62. Beauregard M. Mind does really matter: evidence from neuroimaging studies of emotional self-regulation, psychotherapy, and placebo effect. Prog Neurobiol. 2007;81(4):218-36.

63. Lai CH. Fear network model in panic disorder: the past and the future. Psychiatry Investig. 2019;16(1):16-26.

64. Wisco BE, Sloan DM, Marx BP. Cognitive emotion regulation and written exposure therapy for posttraumatic stress disorder. Clin Psychol Sci. 2013;1(4):435-42.

65. Poletti S, Radaelli D, Cucchi M, Ricci L, Vai B, Smeraldi E, et al. Neural correlates of anxiety sensitivity in panic disorder: a functional magnetic resonance imaging study. Psychiatry Res. 2015;233(2):95-101.

66. Garnefski N, Kraaij V, Spinhoven P. Negative life events, cognitive emotion regulation and emotional problems. Pers Individ Dif. 2001;30(8):1311-27.

67. Strauss AY, Kivity Y, Huppert JD. Emotion regulation strategies in cognitive behavioral therapy for panic disorder. Behav Ther. 2019;50(3):659-71.

68. Koole SL, Webb TL, Sheeran PL. Implicit emotion regulation: feeling better without knowing why. Curr Opin Psychol. 2015;3:6-10.

69. Li H, Wang J, Li C, Xiao Z. (2014) Repetitive transcranial magnetic stimulation (rTMS) for panic disorder in adults. Cochrane Database Syst Rev. 2014;9:CD009083.

\section{Publisher's Note}

Springer Nature remains neutral with regard to jurisdictional claims in published maps and institutional affiliations. 